\title{
Variations of the abductor pollicis longus tendon: an anatomic study
}

\author{
R.A. El-Beshbishy1, 2, G.A. Abdel-Hamid ${ }^{1,3}$ \\ ${ }^{1}$ Department of Anatomy, Faculty of Medicine, King Abdulaziz University, Jeddah, Saudi Arabia \\ 2Department of Anatomy, Faculty of Medicine, Ain Shams University, Cairo, Egypt \\ ${ }^{3}$ Department of Anatomy, Faculty of Medicine, Suez Canal University, Ismaillia, Egypt \\ [Received 2 February 2013; Accepted 29 March 2013]
}

This study was performed to investigate the variations, dimensions and attachment of the abductor pollicis longus (APL) tendon. Fifty upper limbs of adult cadavers were dissected in the Faculty of Medicine, King Abdulaziz University, Jeddah, Saudi Arabia. Variations of APL tendon and its way of insertion were observed; its length, width and thickness were also measured and statistically analysed. No single-tendon-APL was found in all the examined upper limbs. The APL exhibited 2-6 tendons in all the cases. Tendons of APL were classified into medial and lateral. The lateral was considered as the main APL tendon, which was inserted into the base of the first metacarpal bone. The medial tendons were regarded as accessory slips and were inserted most commonly into the base of the first metacarpal bone; $80 \%$ on its anterolateral surface vs. $20 \%$ on the lateral one. The rest of the medial slips extended to trapezium, abductor pollicis brevis, thenar fascia, capsule of first carpometacarpal joint and opponens pollicis, with a frequency of $80 \%$, 60\%, 40\%, 30\% and 20\%, respectively. Transverse tendinous fibres were noted connecting the various slips of the APL tendons. No significant difference was recorded between the length of the main APL tendon and its accessory tendons, however, a highly significant difference was found between their thicknesses as well as their widths. Thus it is verified that the lateral tendon of APL is the main, whereas the medial tendons are accessory. The observations regarding dimensions and variations of the APL and their accessory tendons may prove useful to surgeons as a source of grafting material. (Folia Morphol 2013; 72, 2: 161-166)

Key words: abductor pollicis longus, accessory, tendon, variations, insertions, dimensions

\section{INTRODUCTION}

The comprehensive understanding of variations in tendons of the hand and the fingers is necessary for the proper diagnosis of certain neurological disorders [7]. Among all, the abductor pollicis longus (APL) muscle is one of the most known to present numerous variations. Since there are few reports on such abnormalities in standard anatomy and surgery textbooks, the study of alterations of the APL is of much importance for any academic, surgical and clinical purpose. Variants in the number of tendons of the APL muscle may be asymptomatic and often incidental finding $[7,10,19,23]$.

Moreover, human texts in current use have very little precise information about the frequency of the discrepancies in the distal attachment of the APL

Address for correspondence: Dr. R. El-Beshbishy, Associate Professor of Anatomy, Department of Anatomy, Faculty of Medicine, King Abdulaziz University, Jeddah, Saudi Arabia 42806, tel: +966500588814, fax: +96626400000-23193, e-mail: ranaelbeshbishy@hotmail.com 
tendon. The literature focusing on this subject is meager and a clear description of the tendon insertion is deficient. It was described to be inserted into the base of the first metacarpal bone [24]. The insertion of the APL tendon is adjacent to the carpometacarpal joint of the thumb and assists in stabilising it $[9,12]$. The significance of the thumb in hand function and the complicated surgical procedures used highlighted the clinical importance of anatomical knowledge of such variations [27].

The presence of accessory tendons is an important contributing factor in the development of de Quervain's disease [4]. Duplicated tendons found in the first dorsal compartment of the hand encouraged multiple studies to analyse the anatomy of this area [2]. Recent studies used the accessory tendons of the APL as grafting material for the reconstruction of ruptured extensor pollicis longus tendon [3]. However, to our knowledge, the appropriateness of these tendons for grafting purposes and their dimensions was deficient. Therefore, the present research was performed to investigate the variations in the APL tendons and their dimensions, and to describe their insertion sites.

\section{MATERIALS AND METHODS}

This study was carried out on a total of 50 upper limbs of adult cadavers collected from the dissection room at the Department of Anatomy, Faculty of Medicine, King Abdulaziz University, Jeddah, Saudi Arabia. The muscles of the extensor compartments were dissected; extensor retinaculum was defined and the superficial structures on the lateral part of dorsum of the hand and the thumb were displayed. The extensor retinaculum was split vertically opposite the first extensor compartment and the underlying tendons of APL and extensor pollicis brevis were exposed.

In each specimen, the APL tendon was identified and followed to its insertion. The incidence of variations in its number and sites of attachment were observed. Using a calibrated caliper, the length of the tendon was measured from the point of insertion to the beginning of the muscle belly, whereas the thickness and width were recorded at their insertion sites. The obtained data was tabulated using a 2-tailed Student's t-test and the statistical analysis was performed using SPSS statistical package version 16. The values were considered significant if $p<0.05$.

\section{RESULTS}

The APL muscle was detected in all the dissected upper limbs. In all the specimens, the proximal

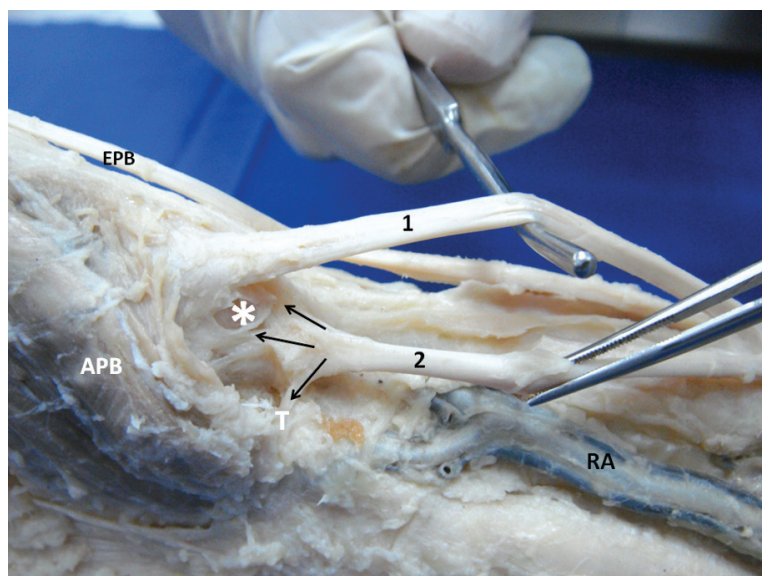

Figure 1. The distal part of right forearm and hand showing two abductor pollicis longus tendons $(1,2)$. The lateral tendon $(1)$ is inserted into the lateral surface of the base of $1^{\text {st }}$ metacarpal bone $\left({ }^{*}\right)$, while the medial tendon (2) gives 3 extensions (arrows) to the lateral and anterolateral surfaces of base of $1^{\text {st }}$ metacarpal bone $\left.{ }^{*}\right)$ and to trapezium (T); APB - abductor pollicis brevis muscle; EPB - extensor pollicis brevis tendon; RA — radial artery.

attachment of the muscle was identified deeply in the middle third of the forearm, taking origin from the middle of the posterior surface of radius, upper most part of the lateral area on the posterior surface of the shaft of ulna and the intervening interosseous membrane. Innervation of the APL was observed in all the specimens to be derived from the branches of the posterior interosseous nerve.

A single tendon of the APL was not found in all the examined upper limbs. The APL was noted to have 2-6 tendons. It existed with 2 tendons in 20 dissected cases (40\%) (10 right side and 10 left side) (Fig. 1), with 3 tendons in 17 dissected cases (34\%) (14 right side and 3 left side) (Fig. 2), with 4 tendons in 9 dissected cases (18\%) (7 right side and 2 left side) (Fig. 3), with 5 tendons in 2 dissected right upper limbs (4\%) (Fig. 4), and 6 tendons in 2 dissected right upper limbs (4\%) (Fig. 5, Table 1). On both sides, all these multiple tendons passed through the first compartment of the extensor retinaculum to reach their distal attachment. In each of these cases, the APL tendons were classified into lateral and medial tendons. The lateral, lying adjacent to the extensor pollicis brevis tendon, was considered the main APL tendon, while the medial tendons were regarded as accessory ones. Transverse tendinous fibres interconnecting some of these tendons were observed (Fig. 3).

The insertion of the APL tendons exhibited several variations. In all the cases, the lateral APL tendons 


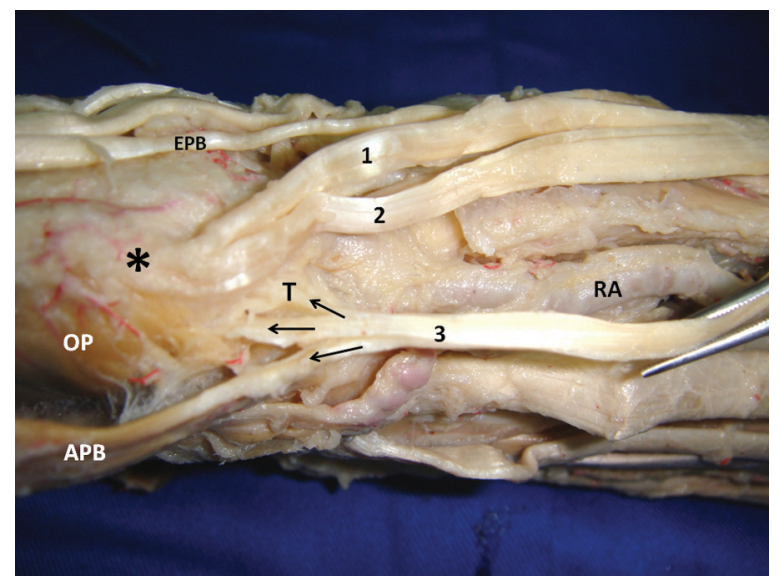

Figure 2. The distal part of right forearm and hand showing three tendons of abductor pollicis longus (1-3). The lateral tendon (1) is inserted into the lateral surface of base of $1^{\text {st }}$ metacarpal bone $\left({ }^{*}\right)$. The medial tendons $(2,3)$; the second tendon (2) is inserted into the lateral surface of base of $1^{\text {st }}$ metacarpal bone and the third one (3) gives 3 extensions (arrows) to trapezium (T), opponens pollicis muscle (OP) and abductor pollicis brevis muscle (APB); EPB - extensor pollicis brevis tendon; RA — radial artery.

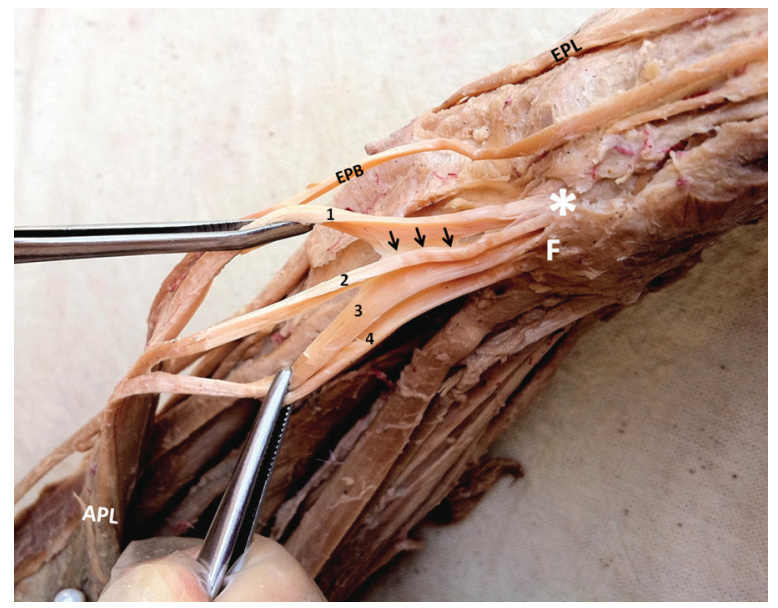

Figure 3. The distal part of left forearm and hand showing four abductor pollicis longus tendons (1-4). Arrows point at the tendinous transverse fibres which interconnect the tendons. The lateral tendon (1) is inserted into the lateral surface of the base of $1^{\text {st }}$ metacarpal bone $\left({ }^{*}\right)$. The medial tendons $(2-4)$; the second tendon (2) is inserted into the lateral surface of base of $1^{\text {st }}$ metacarpal bone $\left({ }^{*}\right)$, while the third and fourth tendons $(3,4)$ are attached to the thenar fascia $(\mathrm{F}) ; \mathrm{APL}$ - abductor pollicis longus muscle; EPB - extensor pollicis brevis tendon; EPL — extensor pollicis longus tendon.

were inserted into the base of the first metacarpal bone; whether to its anterolateral surface (Figs. 4, 5), or its lateral surface (Figs. 1-3). The medial accessory tendons of the APL had different sites of insertions at: the anterolateral surface of base of $1^{\text {st }}$ metacarpal bone (in $80 \%$ ) and its lateral surface (in 20\%) (Figs. 1, 5);

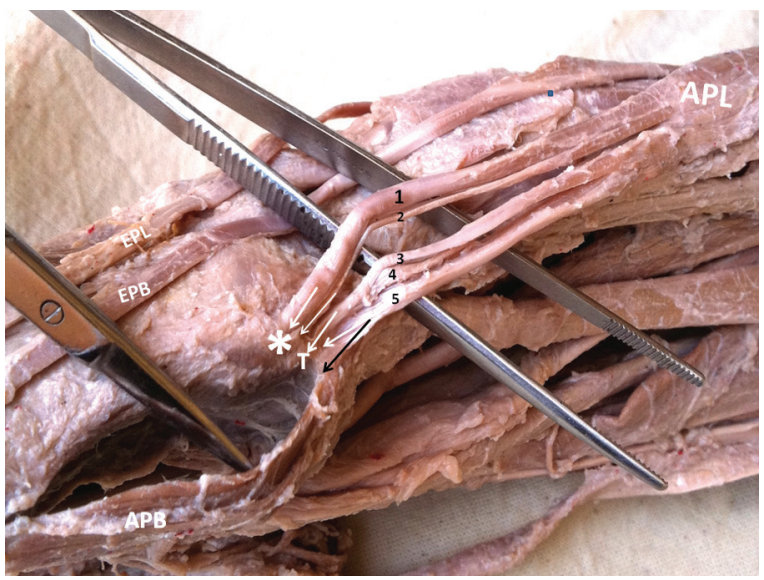

Figure 4. The distal part of right forearm and hand showing five tendons of abductor pollicis longus (1-5). The lateral tendon (1) is inserted into the anterolateral surface of base of $1^{\text {st }}$ metacarpal bone $\left({ }^{*}\right)$. The medial tendons $(2-5)$; the second tendon $(2)$ is inserted into the anterolateral surface of base of $1^{\text {st }}$ metacarpal bone $(*)$, while the third and fourth ones $(3,4)$ are inserted into trapezium (T). The most medial tendon (5) gives 2 slips which are inserted into trapezium (T) and abductor pollicis brevis muscle (APB). $\mathrm{APL}$ - abductor pollicis longus muscle; EPB — extensor pollicis brevis tendon; EPL - extensor pollicis longus tendon.

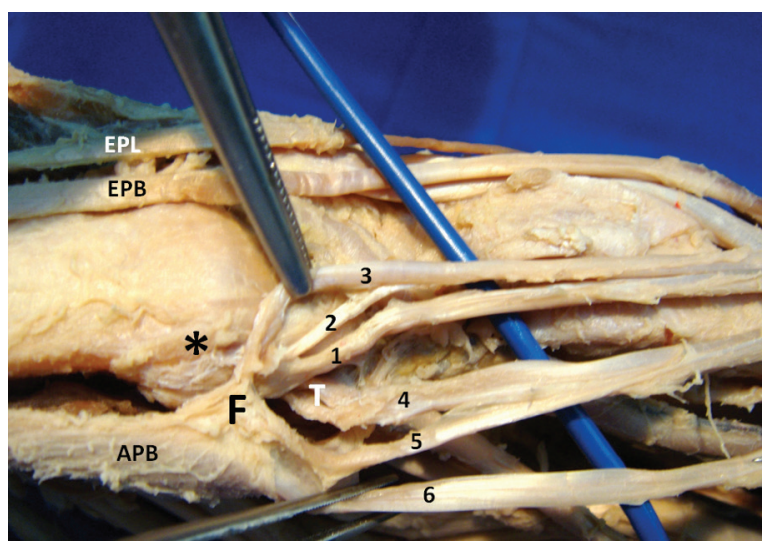

Figure 5. The distal part of right forearm and hand showing six abductor pollicis longus tendons (1-6). The lateral tendon (1) is inserted into the anterolateral surface of the base of $1^{\text {st }}$ metacarpal bone $\left({ }^{*}\right)$. Tendons $(2,3)$ are displayed laterally by forceps to expose the insertions of the medial tendons (2-6). The second tendon (2) is inserted into the anterolateral surface of the base of $1^{\text {st }}$ metacarpal bone $\left({ }^{*}\right)$, while the third (3) and fifth (5) ones are attached to the thenar fascia $(\mathrm{F})$. Tendon (4) is inserted into the trapezium (T). Tendon (6) is inserted into the abductor pollicis brevis muscle (APB); EPB - extensor pollicis brevis tendon; EPL — extensor pollicis longus tendon.

trapezium (in 80\%) (Figs. 1, 2, 4, 5); abductor pollicis brevis (in 60\%) (Figs. 2, 4, 5); thenar fascia (in 40\%) (Figs. 3, 5); capsule of carpometacarpal joint of thumb (in 30\%) or opponens pollicis (in 20\%) (Fig. 2, Table 2).

The mean length of the lateral tendon of APL was $45.2 \pm 15.4 \mathrm{~mm}$, while that of the medial accessory 
Table 1. Variation of the number of abductor pollicis longus (APL) tendons in 50 limbs

\begin{tabular}{lcccc}
\hline \multirow{2}{*}{ No. of APL tendons } & \multicolumn{4}{c}{ No. of upper limbs dissected } \\
\cline { 2 - 5 } & Right & Left & Total & Per cent \\
\hline 1 & 0 & 0 & 0 & $0 \%$ \\
2 & 10 & 10 & 20 & $40 \%$ \\
3 & 14 & 3 & 17 & $34 \%$ \\
4 & 7 & 2 & 9 & $18 \%$ \\
5 & 2 & 0 & 2 & $4 \%$ \\
6 & 2 & 0 & 2 & $4 \%$ \\
\hline
\end{tabular}

Table 2. Variation of insertion of the medial tendons of abductor pollicis longus in 50 limbs

\begin{tabular}{llcc}
\hline Insertion of medial tendons & No. of upper limbs & Per cent \\
\hline Base of $1^{\text {st }}$ metacarpal bone & Anterolateral surface & 40 & $80 \%$ \\
& Lateral surface & 10 & $20 \%$ \\
Trapezium & 40 & $80 \%$ \\
Abductor pollicis brevis & 30 & $60 \%$ \\
Thenar fascia & 20 & $40 \%$ \\
Capsule of carpometacarpal joint of thumb & 15 & $30 \%$ \\
Opponens pollicis & 10 & $20 \%$ \\
\hline
\end{tabular}

Table 3. Length, width and thickness of abductor pollicis longus tendons in 50 limbs; ${ }^{*} p \leq 0.001$

\begin{tabular}{lccc}
\hline & Length $[\mathrm{mm}]$ & Width $[\mathrm{mm}]$ & Thickness $[\mathrm{mm}]$ \\
\hline Lateral tendon & $45.2 \pm 15.4$ & $2.7 \pm 1.07$ & $2.2 \pm 0.5$ \\
Medial tendons & $51.3 \pm 19.9$ & $1.8 \pm 0.7^{*}$ & $1.6 \pm 0.6^{*}$ \\
\hline
\end{tabular}

tendons was $51.3 \pm 19.9 \mathrm{~mm}$. No statistically significant difference was found between both values. The width of the lateral APL tendon was $2.7 \pm 1.07 \mathrm{~mm}$, whereas, that of the accessory ones was $1.8 \pm 0.7 \mathrm{~mm}$. A statistically highly significant difference was found between them ( $p \leq 0.001)$. The thickness of the accessory tendons $(1.6 \pm 0.6 \mathrm{~mm})$ was less than that of the lateral APL tendon $(2.2 \pm 0.5 \mathrm{~mm})$. This difference of thickness was found to be statistically highly significant $(p \leq 0.001)$ (Table 3 ).

\section{DISCUSSION}

In the present study, a single tendon of APL was not recorded in all the examined upper limbs. Also, Coleman et al. [6] declared that a single tendon of APL is odd in 175 examined specimens. However,
Vollala [27] detected a single tendon in $30 \%$ of 100 specimens studied. A varying number of tendon slips of the APL, ranging from 2 to 6 , was observed in all the cases of the current work. Double tendons were the most frequently found in $40 \%$ of the specimens. In accordance, double tendons of APL have been widely documented in $85-98 \%$ of the previous studies $[1,2,11,14,28-30]$. However, this duplication was recorded only in $50 \%$ of the cases $[13,27]$.

In the present investigation, triple tendons were noted in $34 \%$ of the specimens. This triplication was observed by Bravo et al. [2] in 8\% only and by Gonzalez et al. [8] in 13.6\%. Although 4 tendons were found in $18 \%$ of specimens of the current work, it was observed only in 4\% of the cases dissected by Bravo et al. [2] and merely as a case report by Mehta et al. [16]. 
Five and 6 tendons were detected in $8 \%$ of the current specimens, $4 \%$ each. Recently, case reports discovered 5 tendons for APL [21], 6 tendons [18], 7 tendons [17] and 9 tendons [15]. The previous data indicates that it is rare to observe the APL without accessory tendons.

Nayak et al. [18] and Mehta et al. [16] presumed an embryological basis for the accessory APL tendons. The differentiating APL tendon is divided into 3 slips attached to trapezium, first metacarpal and opponens pollicis. Subsequently, new attachments may develop to adjacent abductor pollicis brevis, whereas the link with the opponens pollicis disappears. In the current research, this could be explained by the presence of transverse tendinous fibres connecting the various APL tendons. Also, Van Oudenaarde [25] detected a link by a web of synovium between the main and the accessory APL tendons, with the presence of a bursa between them.

In agreement with Mehta et al. [16], the APL tendons were classified in the present study into lateral (main) and medial (accessory) ones. In 100\% of the cases, both lateral and medial tendons were found to be inserted into the base of the first metacarpal bone. In addition, the medial tendons were also inserted into trapezium in $80 \%$ of the cases. This observation is in accordance with Standring [22], who described the base of the first metacarpal bone and trapezium as the normal sites of the APL insertion. However, Joshi and Joshi [11] and also Vollala [27] recorded the attachment of the accessory tendons to trapezium in only $30 \%$ of the cases. The present study suggests that the insertion of the accessory tendons into both the base of $1^{\text {st }}$ metacarpal bone and trapezium stabilises the carpometacarpal joint of the thumb. Also, the insertion of these tendons to the capsule of the carpometacarpal joint observed in this study adds to this stability. Mehta et al. [16] emphasised that the additional sites of insertion of the APL tendons decrease the liability to chronic dislocation of this joint.

In the present investigation, the medial accessory tendons were also attached to abductor pollicis brevis in $60 \%$ of the specimens. On the contrary, Bravo et al. [2] found the later insertion in $44 \%$ and Vollala [27] in $22 \%$. The present study as well as Joshi and Joshi [11] reported similar percentage (40\%) of attachment of the APL to the thenar fascia; nevertheless, Vollala [27] recorded it in $10 \%$ of the cases only.
Van Oudenaarde and Oostendorp [26] and also Zancolli and Cozzi [30] classified the muscular components of the APL into deep and superficial ones. The deep portion is more proximal and has multiple sites of insertion around the trapezio-metacarpal joint. The superficial portion is more distal and inserts at the base of the first metacarpal. According to the insertions of these portions, the lateral tendon of the APL demonstrated in the current study, corresponds to the superficial portion, while the medial accessory tendons correspond to the deep portions.

In the present study, no statistically significant difference was recorded between the length of the lateral and medial (accessory) APL tendons. Although, Bravo et al. [2] considered the accessory slips of the APL tendon as real tendons due to the absence of differences in their dimensions, a highly statistically significant difference was found between their widths as well as their thicknesses in the present study. It is obvious that the accessory tendons, being less in width and thickness can be chosen in reconstructive surgery of the hand as in cases of osteoarthrosis of the first carpometacarpal joint [20]. Also, it can be used as a tendon graft to restore extension of the thumb $[5,15]$. Further studies are mandatory to define the benefit of using these additional APL tendons as a source of grafting material.

\section{CONCLUSIONS}

In hand surgery, a prior anatomical knowledge of the presence of accessory APL tendons would be useful to avoid any unintended injury. These accessory tendons can be detected by preoperative ultrasound or magnetic resonance imaging and can be functionally useful to compensate the damaged ones.

\section{ACKNOWLEDGEMENTS}

This paper was funded by the Deanship of Scientific Research (DSR), King Abdulaziz University, Jeddah, under grant No. (4-140-D1432). The authors therefore acknowledge with thanks DSR technical and financial support.

\section{REFERENCES}

1. Baba MA (1954) The accessory tendon of the abductor pollicis longus muscle. Anat Rec, 119: 541-547.

2. Bravo E, Barco R, Bullón A (2010) Anatomic study of the abductor pollicis longus: a source for grafting material of the hand. Clin Orthop Relat Res, 468: 1305-1309. 
3. Bullón A, Bravo E, Zarbahsh S, Barco R (2007) Reconstruction after chronic extensor pollicis longus ruptures: a new technique. Clin Orthop Relat Res, 462: 93-98.

4. Bunnel S (1948) Surgery of the hand. $2^{\text {nd }}$ Ed. J.B. Lippincott, Philadelphia.

5. Chitnis SL, Evans DM (1993) Tendon transfer to restore extension of the thumb using abductor pollicis longus. $\mathrm{J}$ Hand Surg Br, 18: 234-238.

6. Coleman SS, Mcafee DK, Anson BJ (1953) The insertion of the abductor pollicis longus muscle; an anatomical study of 175 specimens. Q Bull Northwest Univ Med Sch, 27: 117-122.

7. Giles KW (1960) Anatomical variations affecting the surgery of de Quervain's disease. J Bone Joint Surg Br, 42: 352-355.

8. Gonzalez MH, Sohlberg R, Brown A, Weinzweig N (1995) The first dorsal extensor compartment: an anatomic study. J Hand Surg Am, 20: 657-660.

9. Imaeda T, An KN, Cooney WP $3^{\text {rd }}$ (1992) Functional anatomy and biomechanics of the thumb. Hand Clinic, 8: 9-15.

10. Jackson WT, Viegas SF, Coon TM, Stimpson KD, Frogameni AD, Simpson JM (1986) Anatomical variations in the first extensor compartment of the wrist. A clinical and anatomical study. J Bone Joint Surg Am, 68: 923-926.

11. Joshi SS, Joshi SD (2002) Applied significance of variations of the first extensor compartment of wrist. J Anat Soc India, 51: 159-161.

12. Kauer JM (1987) Functional anatomy of the carpometacarpal joint of the thumb. Clin Orthop Relat Res, 220: 7-13.

13. Khoury Z, Bertelli J, Gilbert A (1991) The subtendons of the abductor pollicis longus muscle. Surg Radiol Anat, 13: $245-246$.

14. Lacey T II, Goldstein LA, Tobin CE (1951) Anatomical and clinical study of the variations in the insertions of abductor pollicis longus tendon associated with stenosing tenovaginitis. J Bone Jt Surg Am, 33: 347-350.

15. Mansur DI, Krishnamurthy A, Nayak SR, Kumar CG, Rai R, Sujatha D'Costa S, Prabhu LV (2010) Multiple tendons of abductor pollicis longus. Int J Anat Variations, 3: 25-26.

16. Mehta V, Arora J, Suri RK, Rath G (2009) A rare quadruplicate arrangement of abductor pollicis longus tendons: anatomical and clinical relevance. Clinics (Sao Paulo), 64: 153-155.
17. Melling M, Wilde J, Schnallinger M, Schweighart W, Panholzer M (1996) Supernumerary tendons of the abductor pollicis. Acta Anat (Basel), 155: 291-294.

18. Nayak SR, Krishnamurthy A, Pai MM, Prabhu LV, Ramanathan LA, Ganesh Kumar C, Thomas MM (2008) Multiple variations of the extensor tendons of the forearm. Rom J Morphol Embryol, 49: 97-100.

19. Paul S, Das S (2007) Variant abductor pollicis longus muscle: a case report. Acta Medica, 50: 213-215.

20. Saehle T, Sande S, Finsen V (2002) Abductor pollicis longus tendon interposition for arthrosis in the first carpometacarpal joint: 55 thumbs reviewed after $3(1-5)$ years. Acta Orthop Scand, 73: 674-677.

21. Schulz CU, Anetzberger $\mathrm{H}$, Pfahler M, Maier M, Refior $\mathrm{HJ}$ (2002) The relation between primary osteoarthritis of the trapeziometacarpal joint and supernumerary slips of the abductor pollicis longus tendon. J Hand Surg $\mathrm{Br}$, 27: 238-241.

22. Standring $S$ (2008) Gray's anatomy. The anatomical basis of clinical practice. 40th Ed. Elsevier, Churchill Livingstone, London.

23. Stein AH Jr (1951) Variations of the tendons of insertion of the abductor pollicis longus and extensor pollicis brevis. Anat Rec, 110: 49-55.

24. Tountas CP, Bergman RA (1993) Anatomic variations of the upper extremity. Churchill Livingstone, New York, pp. 126-132.

25. Van Oudenaarde E (1991) Structure and function of the abductor pollicis longus muscle. J Anat, 174: 221-227.

26. Van Oudenaarde E, Oostendorp R (1992) Significance of the innervation pattern of the human abductor pollicis longus muscle. J Anat, 181: 155-159.

27. Vollala VR (2006) Abductor pollicis longus: A study of 50 south Indian cadavers. Firat Tip Dergisi, 12: 17-19.

28. Warwick R, Williams $P$ (1973) Gray's anatomy. $35^{\text {th }}$ Ed. Edinburgh, Longman.

29. Zancolli EA (2001) The trapeziometacarpal joint. Tenotomy of the accessory tendons in early osteoarthritis. Hand Clin, 17: 13-43.

30. Zancolli EA, Cozzi EP (1993) Thumb muscles. Atlas de anatomia quirurgica de la mano. Ed 1. Medica Panamericana, Madrid. 